\title{
Microbiology in space
}

The launching of artificial earth satellites, space shuttles and rockets has now opened new avenues in almost all branches of knowledge. One such exceptionally interesting area which got huge impetus from such developments in space technology is the area of 'space microbiology'. Aim of this discipline is to study the biological responses of microorganisms in response to the harsh and inhospitable conditions (intense solar UV radiation, space vacuum, thermal extremes and microgravity) of outer space environment and also to use microbes for studying the conditions of life there. The 'spaceflight microbes' hold great potential for the development of novel therapeutics and vaccines against infectious diseases. Moreover, for the manned spaceflight missions, understanding human biological changes and microbial responses while living in the closed quarters in space is important to the health, safety and performance of crewmembers. For instance, prolonged exposure to cosmic radiation and microgravity is believed to have a negative effect on the human immune system; hence, microorganisms have been studied as radiobiological model systems in space for assessing radiation risks to humans in space. Once the microbes return from space, their responses to selected space parameters like varying gravity conditions are studied and compared with those obtained on ground. In order to carry out these microbiological studies in space, special facilities simulating the conditions of outer space like microgravity or 'weightlessness' are used. A wide variety of payloads have been developed by numerous international teams like National Aeronautics and Space Administration (NASA) and European Space Agency (ESA) to carry out cellular and molecular biology studies inside the pressurized environment of the spacecraft. With this beginning, the space research is increasingly booming and is likely to provide further momentum towards commercial pharmaceutical applications like secondary metabolite (antibiotic) production, controlling the spread of multidrug resistant pathogens and most importantly vaccine development. So, it would be appropriate to mention that all the experimental data obtained from such space studies can serve as a 'time-machine' for predicting the feasibility as well as sensitivity of life in the space.
Editor-in-Chief Prof. Rup Lal 